\title{
Periostin Contributes to Cisplatin Resistance in Human Non-Small Cell Lung Cancer A549 Cells via Activation of Stat3 and Akt and Upregulation of Survivin
}

\author{
Wenxia Hua Pule Jin ${ }^{\mathrm{a}}$ Wei Liu ${ }^{\mathrm{b}}$ \\ aDepartment of Respiratory Medicine, the Fourth Hospital of Hebei Medical University, Shijiazhuang,

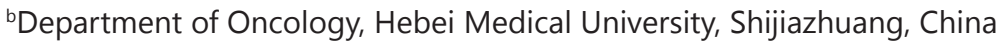

\section{Key Words}

Chemoresistance • Lung cancer • Periostin • Stat3 signaling • Therapeutic target

\begin{abstract}
Background/Aims: Periostin is upregulated in non-small cell lung cancer (NSCLC). This study was done to explore the function of periostin in the development of cisplatin (CDDP) resistance in NSCLC. Methods: The effects of overexpression or knockdown of periostin on CDDP sensitivity was examined in A549 cells. The involvement of signal transducer and activator of transcription 3 (Stat3) and Akt signaling in the action of periostin was checked. The in vivo effect of periostin silencing on CDDP susceptibility was determined in a mouse xenograft model. Results: Periostin was significantly upregulated in CDDP-resistant A549 cells, compared to parental controls. Overexpression of periostin rendered A549 cells more resistant to CDDP-induced apoptosis and enhanced Stat3 and Akt phosphorylation and survivin expression. Periostin-mediated protection against CDDP-induced apoptosis was compromised by downregulation of survivin. Furthermore, knockdown of periostin re-sensitized CDDPresistant A549 cells to CDDP. After CDDP treatment, greater volume reduction was observed in periostin-silenced xenograft tumors than in control tumors, which was accompanied by reduced levels of phosphorylated Stat3 and survivin in periostin-depleted tumors. Conclusion: In conclusion, periostin promotes CDDP resistance in NSCLC cells largely through activation of Stat3 and Akt and upregulation of survivin and thus represents a promising target for overcoming CDDP resistance.

\section{Introduction}

Non-small cell lung cancer (NSCLC), accounting for about $80 \%$ of all lung cancer cases, is one of the leading causes of cancer-related mortality in the world [1]. Most NSCLC patients are diagnosed at locally advanced or metastatic disease stage and have a dismal prognosis, 
with the overall 5-year survival rate of 20\% [2]. Cisplatin (CDDP)-based chemotherapy is currently the first-line therapy for non-resectable or advanced NSCLC. However, emergence of chemoresistant tumor cells ultimately leads to therapeutic failure. Drug efflux, enhancement of DNA repair activity, defects in cell death pathways, and activation of prosurvival signaling pathways have been suggested to contribute to CDDP resistance in tumor cells $[3,4]$. Identification of key mediators of CDDP resistance is of importance in improving the efficacy of chemotherapy against NSCLC.

Periostin, originally identified as an osteoblast-specific factor [5], has been found to be overexpressed in various types of human cancers including NSCLC [6]. Periostin plays a critical role in tumor cell survival. Bao et al. [7] reported that periostin confers protection against stress-induced apoptosis in colon cancer cells. In another study by this research group, periostin shows the ability to augment the survival of A549 NSCLC cells under hypoxic conditions [8]. A recent study has shown that periostin induces chemoresistance in colon cancer cells [9]. These studies suggest periostin as a novel survival factor for cancer cells.

Therefore, in this study, we aimed to explore the possible role of periostin in the development of CDDPresistancein NSCLC cells. Signal transducer and activator of transcription 3 (Stat3) signaling is constitutively activated and associated with chemoresistance in NSCLC [10]. Inhibition of Stat3 signaling has been documented to sensitize A549 NSCLC cells to DNA damaging drugs doxorubicin and CDDP [11]. Akt signaling is also involved in the chemoresistance of A549 cells to CDDP $[12,13]$. We thus tested the involvement of Stat 3 and Akt signaling in the activity of periostin.

\section{Materials and Methods}

\section{Cell culture}

Human NSCLC A549 cells (originated from lung adenocarcinoma) were purchased from the Shanghai Cell Bank of Chinese Academy of Sciences (Shanghai, China) and maintained in Dulbecco's modified Eagle's medium (DMEM; Invitrogen, Carlsbad, CA, USA) supplemented with $100 \mu \mathrm{g} / \mathrm{mL}$ penicillin, 100 $\mu \mathrm{g} / \mathrm{mL}$ streptomycin, and $10 \%$ fetal bovine serum (FBS; Invitrogen) at $37^{\circ} \mathrm{C}$ in a humidified atmosphere with $5 \% \mathrm{CO}_{2}$. The CDDP-resistant A549 cell line (A549/CDDP) was established by exposure to increasing concentrations $(0.5,1,4,10$, and $20 \mu \mathrm{M})$ of CDDP (Sigma-Aldrich, St. Louis, MO, USA). At each concentration, A549 cells were allowed to grow for 2 weeks. The CDDP-resistant cells were maintained in the medium containing $10 \mu \mathrm{M}$ of CDDP.

\section{MTT assay}

Cells were seeded in triplicate onto 96-well plates at a density of $5 \times 10^{3}$ cells per well and incubated with different concentrations of CDDP ranging from 0 to $30 \mu \mathrm{M}$ for $48 \mathrm{~h}$. The cell culture was added with 3-(4, 5-dimethylthiazol-2-yl)-2, 5-diphenyltetrazolium bromide (MTT) solution (Sigma-Aldrich) at a final concentration of $0.5 \mathrm{mg} / \mathrm{mL}$ and incubated for additional $4 \mathrm{~h}$ at $37^{\circ} \mathrm{C}$. Dimethyl sulfoxide (DMSO; Sigma-Aldrich) was added to each well to dissolve the formazan product. Absorbance was measured at a wavelength of $570 \mathrm{~nm}$. The concentration-cell viability curve was plotted, from which the half-maximal inhibitory concentration (IC50) was estimated.

\section{Plasmids, short hairpin RNAs (shRNA), and cell transfection}

For construction of a periostin-expressing plasmid [14], full-length human periostin cDNA (OriGene, Rockville, MD, USA) was amplified by PCR and subcloned into pcDNA3.1 (+) vector (Invitrogen). The identity of the resulted plasmid (pcDNA3.1/periostin) was confirmed by DNA sequencing. Periostin- and survivintargeting shRNAs as well as negative control shRNAs were purchased from Santa Cruz Biotechnology (Santa Cruz, CA, USA).

For transfection experiments, cells were seeded onto 6-well plates $\left(5 \times 10^{5}\right.$ cells/well) the day before transfection. Cells were transfected with pcDNA3.1 $(+)$ empty vector $(0.5 \mu \mathrm{g})$, pcDNA3.1/periostin plasmid $(0.5 \mu \mathrm{g})$, or together with survivin or control shRNAs (1 $\mu \mathrm{g}$ for each shRNA) using the Lipofectamine 2000

\section{KARGER}




\section{Cellular Physiology Cell Physiol Biochem 2016;38:1199-1208 \begin{tabular}{l|l} 
and Biochemistry Published online: March 17, 2016 & $\begin{array}{l}\text { DO } 2016 \text { The Author(s). Published by S. Karger AG, Basel } \\
\text { www.karger.com/cpb }\end{array}$
\end{tabular}}

Hu/Jin/Liu: Periostin Contributes to Cisplatin Resistance

transfection reagent (Invitrogen) according to the manufacturer's instructions. At $24 \mathrm{~h}$ after transfection, cells were harvested for gene expression analysis or exposure to CDDP for $48 \mathrm{~h}$ before further assays.

For generation of stable periostin knockdown cells, periostin-specific shRNA or control shRNAs were transfected into A549/CDDP cells. Transfected cells were selected for 2 weeks in the presence of $0.5 \mathrm{mg} /$ mL of G418 (Sigma-Aldrich). G418-resistant cells were pooled and used in the subsequent experiments.

\section{Quantitative real-time PCR ( $q R T-P C R$ ) analysis}

Periostin mRNA levels were measured using qRT-PCR analysis, as described previously [15]. In brief, total cellular RNA was isolated using Trizol reagents (Invitrogen) and reversely transcribed to firststrand cDNA using TaqMan reverse transcription reagents (Applied Biosystems, Foster City, CA, USA). PCR amplifications were carried out on an ABI 7900 TaqMan Sequence Detection System (Applied Biosystems). The sequences of the PCR primers used were as follows: human periostin forward, $5^{\prime}$-GCG CTT TAG CAC CTT CCT-3' and reverse, 5'-GCA CAA ATA ATG TCC AGT CTCC-3'; human glyceraldehyde-3-phosphate dehydrogenase (GAPDH) forward, 5'-CGA CCA CTT TGT CAA GCTCA-3' and reverse, 5'-AGG GGT CTA CAT GGC AACTG-3'. The relative periostin mRNA expression was determined according to the $2^{-\Delta \Delta \mathrm{ct}}$ method after normalization against GAPDH [16].

\section{Western blot analysis}

Cells were lysed in Radio-immuno-protein assay (RIPA) buffer (Thermo Scientific, Rockford, IL, USA) containing Complete Protease Inhibitor Cocktail (Roche, Indianapolis, IN, USA). Protein samples (20 $\mu \mathrm{g}$ per lane) were subjected to sodium dodecyl sulfate-polyacrylamide gel electrophoresis and transferred to nitrocellulose membranes. Membranes were blocked with $5 \%$ non-fat milk in $1 \times$ TBST $(10 \mathrm{mM}$ Tris- $\mathrm{HCl}$, $150 \mathrm{mM} \mathrm{NaCl}, \mathrm{pH} 8.0$, and $0.1 \%$ Tween 20), and incubated with primary antibodies overnight at $4^{\circ} \mathrm{C}$. The primary antibodies included: anti-periostin (sc-67233, Santa Cruz Biotechnology), anti-cleaved caspase-3 (\#9661), anti-phospho-Stat3 (\#9131), anti-Stat3 (\#12640), anti-phospho-Akt (\#9271), anti-Akt (\#9272), anti-survivin (\#2803), and anti- $\beta$-actin (\#8457, Cell Signaling Technology, Danvers, MA, USA). After washing, membranes were incubated with horseradish peroxidase-conjugated secondary antibodies (Santa Cruz Biotechnology). Membranes were developed using a chemiluminescence method (ECL, Amersham Biosciences, Piscataway, NJ, USA). Densitometric analysis of protein signals was conducted with Quantity One software (Bio-Rad Laboratories, Hercules, CA, USA).

\section{Apoptosis detection by flow cytometry}

Cell apoptosis was measured using the Annexin V-FITC Apoptosis Detection Kit (Sigma-Aldrich) according to the manufacturer's protocol. After double staining with Annexin V-FITC and propidium iodide (PI), cells were analyzed using a FACSCalibur flow cytometer (BD Biosciences, Franklin Lakes, NJ, USA).

\section{Animal experiments}

All animal experiments were performed in accordance with the National Institutes of Health Guidelines for the Care and Use of Laboratory Animals and approved by the Institutional Animal Care Committee of Hebei Medical University (Shijiazhuang, China). Female BALB/C nude mice (4- to 6-week-old) were purchased from Beijing University Experimental Animal Center (Beijing, China). Control shRNA- and periostin shRNA-transfected A549/CDDP cells $\left(4 \times 10^{6}\right)$ were subcutaneously injected into the left and right flank of each nude mice, respectively. At 5 days after cell transplantation, the mice (4 mice per treatment group) were randomly assigned to receive an intraperitoneal injection of CDDP ( $5 \mathrm{mg} / \mathrm{kg}$ every 4 days) [17] or PBS. Tumor volume was measured every 5 days. At 30 days after cell transplantation, all mice were sacrificed. The tumors were removed and subjected to Western blot analysis.

\section{Statistical analysis}

Data are presented as means \pm standard deviation. All statistical calculations were carried out using SPSS 11.7 software (SPSS, Chicago, IL, USA). Statistical differences among multiple groups were analyzed by one-way analysis of variance followed by the Tukey test. A $P$-value of $<0.05$ was considered statistically significant. 


\section{Results}

Periostin is upregulated in CDDP-resistant A549 cells

To confirm the development of acquired chemoresistance after stepwise exposure to increasing concentrations of CDDP, A549/CDDP and parental cells were treated with different concentrations of CDDP for $48 \mathrm{~h}$ and cell viability was measured by the MTT assay. As shown in Fig. 1A, the IC ${ }_{50}$ of A549/CDDP and A549 cells for CDDP was about $25 \mu \mathrm{M}$ and 10 $\mu \mathrm{M}$, respectively, indicating that A549/CDDP cells were more resistant to CDDP than A549 cells.

qRT-PCR analysis revealed that the expression level of periostin mRNA was approximately 6 -fold greater in A549/CDDP cells than in A549 cells $(P<0.05$; Fig. 1B). The induction of periostin expression in CDDP-resistant cells was confirmed at the protein level by Western blot analysis (Fig. 1C).

Periostin decreases the sensitivity of A549 cells to CDDP

Next, we sought to check if the u-pregulation of periostin affected the sensitivity of A549 cells to CDDP. As shown in Fig. 2A, transfection of periostin-expressing plasmid led to overexpression of periostin in A549 cells. After incubation with different concentrations of CDDP, periostin-overexpressing cells were more viable than empty vector-transfected cells $(P<0.05$; Fig. 2B).

To further determine the effect of periostin on the sensitivity of A549 cells to CDDP, annexin-V/PI staining assay was performed to measure the apoptotic response after treatment with 10 or $30 \mu \mathrm{M}$ CDDP for $48 \mathrm{~h}$. As shown in Fig. 2C, periostin overexpression significantly $(P<0.05)$ decreased CDDP-induced apoptosis, compared with the control group. The apoptosis rate after treatment with $30 \mu \mathrm{M}$ CDDP was $26.1 \pm 1.2 \%$ and $9.5 \pm$ $0.7 \%$ in control and periostin-overexpressing cells, respectively (Fig. 2C). The protection

Fig. 1. Periostin is upregulated in CDDP-resistant A549 cells (A549/CDDP). (A) A549 cells and A549/CDDP cells were treated with increasing concentrations of CDDP for $48 \mathrm{~h}$. Cell viability was determined by the MTT assay. The (B) mRNA and (C) protein levels of periostin were analyzed by qRT-PCR and Western blot analysis, respectively. Data are representative of three independent experiments performed in triplicates. ${ }^{*} P<0.05$ vs. parental A549 cells.

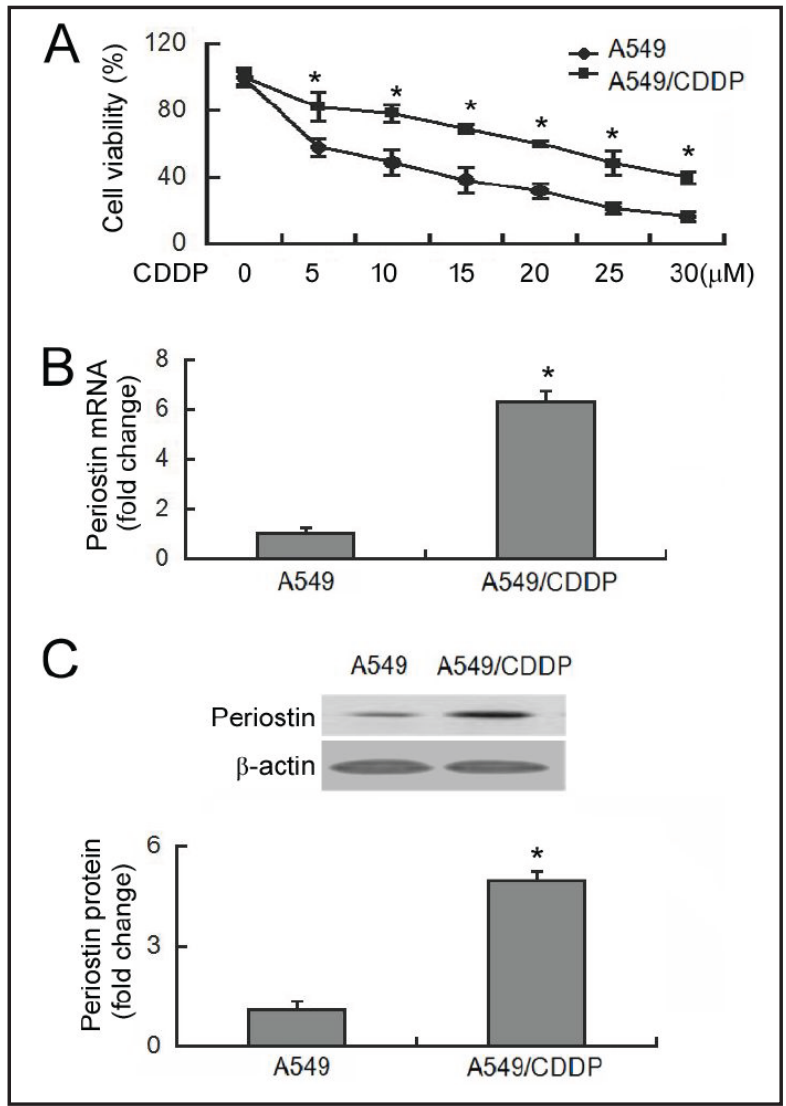


Fig. 2. Periostin overexpression attenuates the sensitivity of A549 cells to CDDP. (A) Western blot analysis of periostin protein in A549 cells transfected with pcDNA3.1(+) vector or pcDNA3.1/periostin. (B) A549 cells transfected with pcDNA3.1(+) vector or pcDNA3.1/periostin were treated with indicated concentrations of CDDP and tested for the viability using the MTT assay. (C) Cell apoptosis were measured by flow cytometry after Annexin V/PI double staining. (D) Western blot analysis of cleaved (active) caspase-3 levels. Bar graphs show data from three independent experiments performed in triplicates. ${ }^{*} P<0.05$.

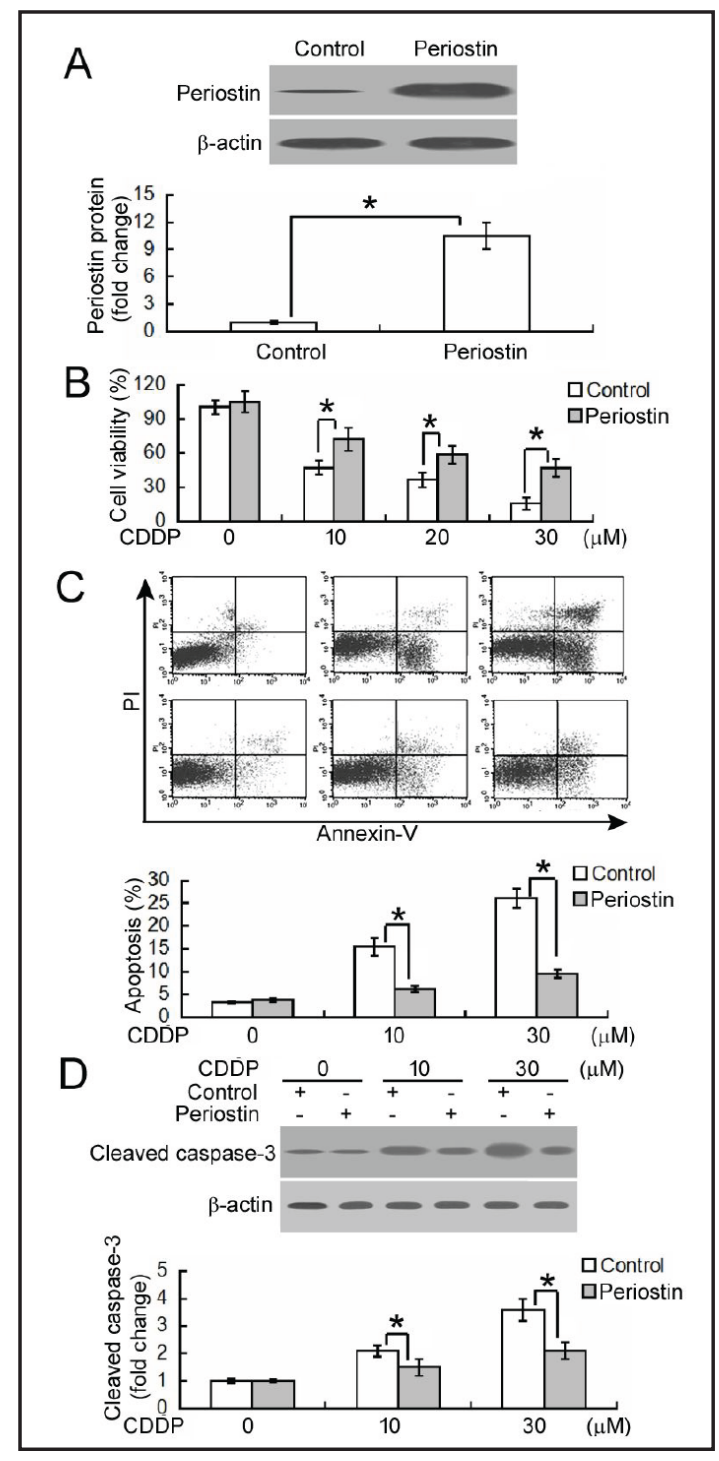

against CDDP-induced apoptosis by overexpression of periostin was also confirmed in an independent NSCLC cell line, H460 (data not shown). In addition, Western blot analysis was done to analyze caspase-3 cleavage, another indicator of apoptosis. We found that the levels of cleaved caspase-3 were significantly $(P<0.05)$ increased in parental A549 cells after CDDP treatment (Fig. 2D). Periostin overexpression significantly prevented the induction of cleaved caspase-3 by CDDP.

Periostin-mediated CDDP resistance is linked to activation of Stat3 and Akt and upregulation of survivin

Next, we tested the signaling pathways involved in periostin-mediated CDDP resistance. Western blot analysis showed that periostin overexpression did not affect the protein level of total Stat3 or Akt (Fig. 3A). However, Stat3 and Akt phosphorylation was enhanced by ectopic expression of periostin, irrespective of the presence or absence of CDDP (Fig. 3A). Stat3 activation has been shown to confer apoptosis resistance via regulation of survivin expression [18]. We found that CDDP treatment of A549 parental cells significantly reduced the level of survivin, compared to untreated cells (Fig. 3A). However, in periostinoverexpressing A549 cells, the level of survivin was elevated after CDDP treatment.

To examine the role of survivin in periostin-mediated apoptosis resistance to CDDP, we co-transfected A549 cells with periostin- and survivin shRNA-expressing plasmids before KARGER 
Fig. 3. Periostin-mediated CDDP resistance is linked to activation of Stat3/survivin signaling. A549 cells were transfected with pcDNA3.1 (+) vector or pcDNA3.1/periostin $24 \mathrm{~h}$ before CDDP treatment. Indicated proteins were examined by Western blot analysis. (B and C) A549 cells were transfected with pcDNA3.1(+) empty vector, or pcDNA3.1/periostin, together with survivin (S-shRNA) or control shRNAs (C-shRNA). At $24 \mathrm{~h}$ after transfection, cells were exposed to CDDP for $48 \mathrm{~h}$. (B) Cell apoptosis and (C) cleaved caspase-3 level were determined by Annexin-V/PI staining and Western blot analysis, respectively. Bar graphs show data from three independent experiments performed in triplicates. ${ }^{*} P<0.05$.

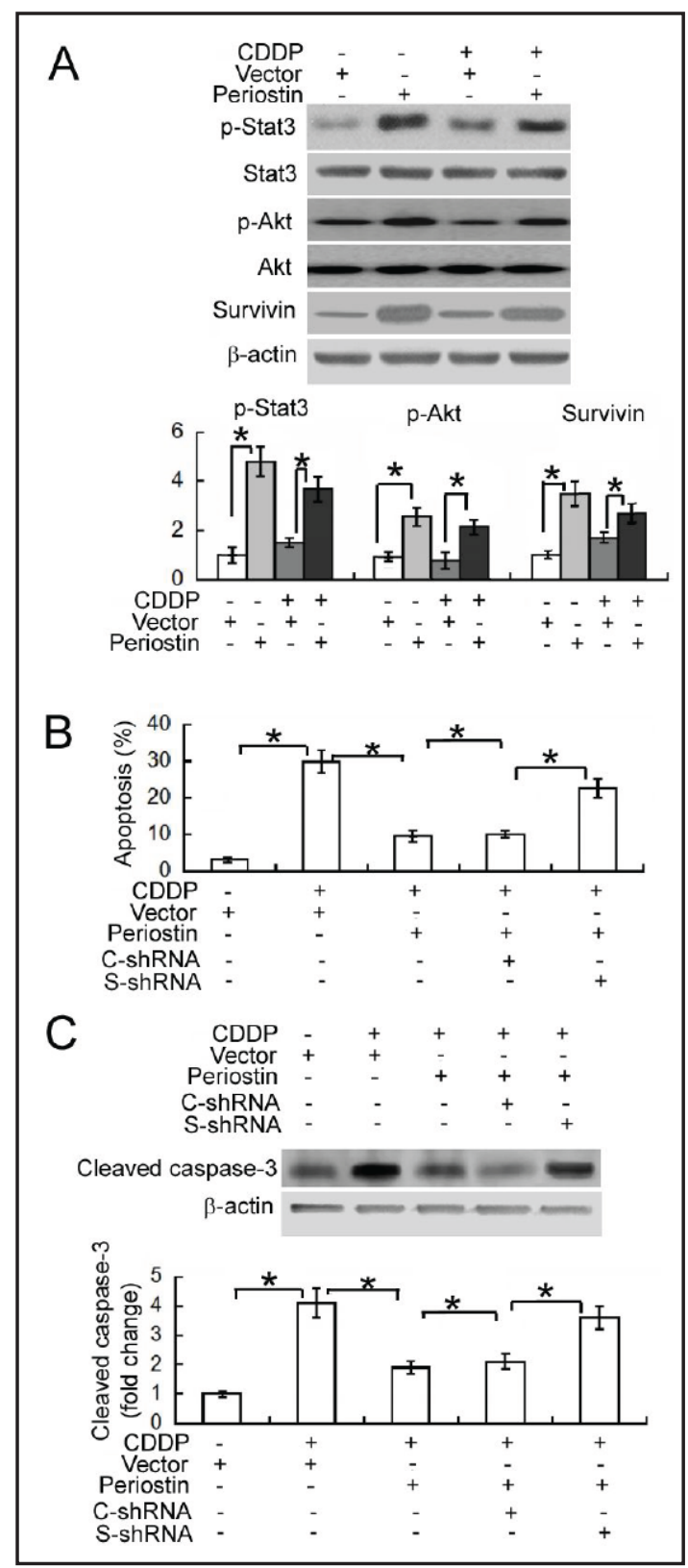

exposure to CDDP and examined apoptosis response. As shown in Fig. 3B, co-transfection of survivin shRNA interfered with the antiapoptotic activity of periostin, partially restoring the apoptosis response to CDDP. Additionally, periostin-mediated inhibition of caspase-3 cleavage in CDDP-treated cells was reversed by co-transfection of survivin shRNA (Fig. 3C).

Periostin silencing re-sensitizes CDDP-resistant A549 cells

Having identified the contribution of periostin in CDDP resistance, we next checked whether targeting periostin could re-sensitize CDDP-resistant cells to this drug. As shown in Fig. 4A, shRNA-mediated silencing of periostin significantly decreased the viability of A549/ CDDP cells after exposure to 10 or $30 \mu \mathrm{M}$ CDDP, compared to control shRNA-transfected cells. Consistently, periostin downregulation restored a significant apoptotic response to CDDP (Fig. 4B). Mouse studies were performed to validate the effect of periostin depletion on CDDP sensitization in vivo. As shown in Fig. 4C, periostin-silenced xenograft tumors grew slowly than control tumors. At the end of the experiment, CDDP treatment significantly reduced the 
Fig. 4. Periostin silencing re-sensitizes CDDP-resistant A549 cells. A549/CDDP Cells were transfected with periostin (P-shRNA) or control shRNAs (C-shRNA) 24 before exposure to CDDP for another 48 h. (A) Cell viability was determined by the MTT assay and (B) apoptosis was measured by flow cytometry after annexin-V/PI staining. Bar graphs show data from three independent experiments performed in triplicates. ${ }^{*} P<0.05$. (C and D) C-shRNA- and P-shRNA-transfected A549/CDDP cells $\left(4 \times 10^{6}\right)$ were subcutaneously injected into the left and right flank of each nude mice, respectively. At 5 days after cell transplantation, the mice (4 mice per group) were randomly assigned to receive an intraperitoneal injection of CDDP or PBS. (C) Tumor volume curves were plotted against days after cell injection. ${ }^{*} P<$ $0.05(n=4)$. (D) At 30 days after cell transplantation, all mice were sacrificed, and tumors were excised and subjected to Western blot analysis. Representative Western blots are shown.

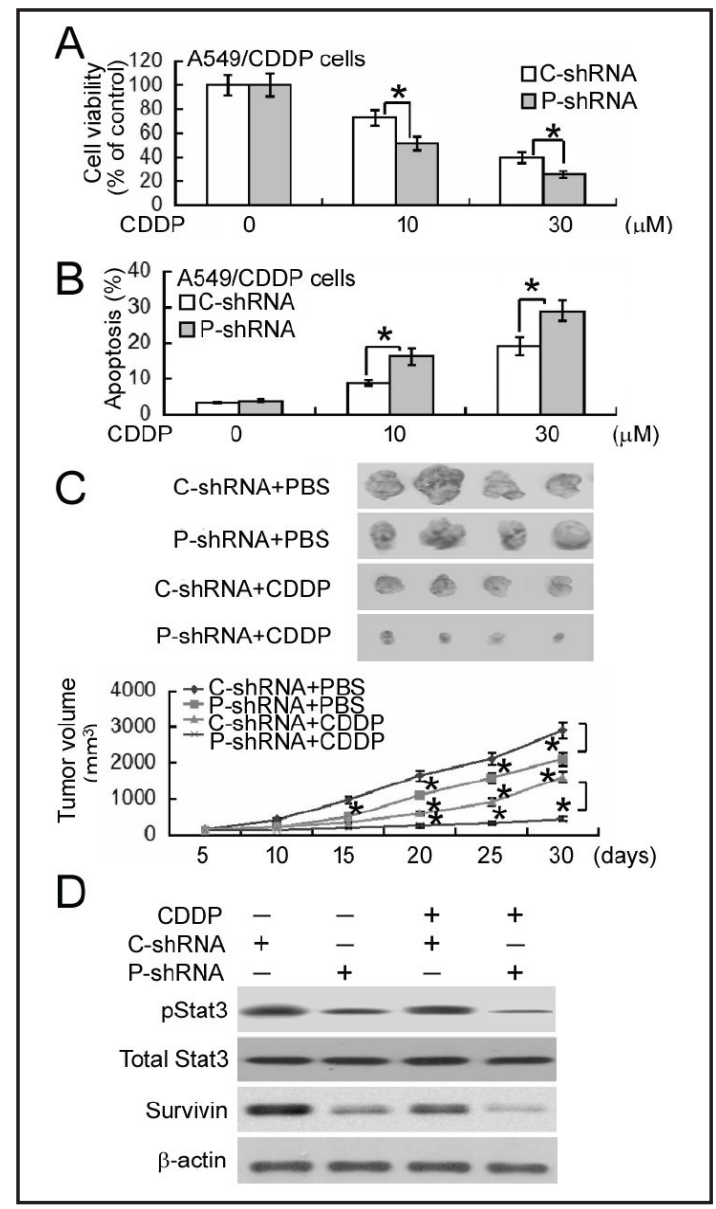

volumes of control and periostin-silenced tumors to $56 \%$ and $21 \%$ of corresponding PBStreated tumors, respectively $(P<0.05)$. Western blot analysis of resected xenograft tumors demonstrated that after CDDP treatment, periostin-depleted tumors expressed lower levels of phosphorylated Stat3 and survivin and higher amounts of cleaved caspase-3 than control tumors (Fig. 4D). These results indicate that periostin depletion re-sensitizes CDDP-resistant A549 cells to CDDP treatment and suppresses the activation of Stat3/survivin signaling in vivo.

\section{Discussion}

Although an initial response occurs after CDDP-based chemotherapy, many NSCLC patients will eventually develop resistance to such treatments [19]. To identify key mediators of CDDP resistance in NSCLC, we established a CDDP-resistant A549 cell model via stepwise exposure to increasing concentrations $(0.5$ to $20 \mu \mathrm{M})$ of CDDP. This model mimics clinical conditions under which NSCLC cells develop CDDP resistance.

Periostin has been identified as a hypoxia responsive gene in different cellular contexts such as pulmonary arterial smooth muscle cells [20] and NSCLC cells [8]. This gene shows the ability to promote the survival of cancer cells under hypoxic conditions [8]. Compelling evidence indicates that hypoxia is causally linked to chemoresistance in NSCLC. Schnitzer et al. [21] reported that hypoxia-induced upregulation of sphingosine kinase 2 contributes to chemoresistance in A549 cells via increased synthesis and release of sphingosine-1phosphate, a bioactive lipid. Therefore, in this study, we sought to determine the role of CDDP resistance in NSCLC cells. Our data showed that periostin was significantly upregulated in 


\section{Cellular Physiology Cell Physiol Biochem 2016;38:1199-1208 \\ \begin{tabular}{l|l} 
and Biochemistry Published online: March 17, 2016 & $\begin{array}{l}\text { (c) 2016 The Author(s). Published by S. Karger AG, Basel } \\
\text { www.karger.com/cpb }\end{array}$
\end{tabular} \\ Hu/Jin/Liu: Periostin Contributes to Cisplatin Resistance}

CDDP-resistant A549 cells, suggesting its possible involvement in the acquisition of drug resistance. Chemotherapeutic drug-induced periostin expression has also been observed in colon cancer cells [9]. To test the hypothesis that upregulation of periostin may contribute to cell survival after exposure to chemotherapeutic drugs, we overexpressed periostin in A549 cells and measured changes in cell viability and apoptosis in response to CDDP treatment. We found that overexpression of periostin rendered A549 cells more resistant to CDDP, as evidenced by increased cell viability and reduced apoptotic response. Caspase- 3 is a key mediator of the apoptosis pathway induced by CDDP [22]. Notably, ectopic expression of periostin inhibited CDDP-induced expression of active (cleaved) caspase-3 in A549 cells, suggesting that the anti-apoptotic effect of periostin involves prevention of caspase- 3 activation. In agreement with our results, periostin has been previously reported to impair chemotherapeutic drug-induced activation of caspase- 3 and apoptotic response in gastric cancer cells [23]. Taken together, our data highlight the importance of periostin in CDDP resistance of NSCLC cells, which is ascribed to its anti-apoptotic activity.

Several molecular pathways are involved in different functional aspects of periostin $[24,25]$. Zhang et al. [24] demonstrated that periostin facilitates angiogenesis in keloids via activation of the ERK 1/2 and focal adhesion kinase (FAK) pathways. Yang et al. [25] reported that periostin promotes skin sclerosis through a PI3K/Akt-dependent mechanism in a mouse model of scleroderma. Activation of the Akt/PKB pathway has been shown to mediate the enhancement of colon cancer metastasis by periostin [7]. Stat3 signaling is implicated in chemoresistance of NSCLC cells and represents an important therapeutic target for NSCLC [10]. Pharmacological inhibition of the Jak/Stat pathway has been documented to overcome CDDP resistance in NSCLC [26]. Therefore, in this work, we investigated whether activation of Stat3 signaling was linked to periostin-mediated CDDP resistance. We showed that periostin overexpression induced the activation of Stat3 and Akt and the expression of survivin in A549 cells, even in the presence of CDDP. Moreover, depletion of survivin significantly attenuated the protective effect of periostin against CDDP cytotoxicity, as evidenced by increased apoptotic response and caspase- 3 activation. Collectively, periostin confers protection against CDDP-induced apoptosis in NSCLC cells largely through activation of Stat3 and Akt and upregulation of survivin.

Inhibition of periostin has been documented to suppress tumor growth and metastasis in many cancers [27, 28]. For instance, Zhu et al. [27] reported that blockade of periostin activity using neutralizing monoclonal antibody results in reduced ovarian tumor growth and metastasis. Our in vitro and in vivo studies further confirmed the significance of periostin as a therapeutic target in chemoresistant NSCLC. We found that downregulation of periostin significantly re-sensitized A549/CDDP cells to CDDP treatment. In a mouse model, CDDP treatment yielded greater tumor volume reduction in periostin-silenced A549/CDDP xenograft tumors than in control tumors. Western blot analysis confirmed that the chemosensitizing effect of periostin silencing was associated with suppression of Stat3 activation and survivin expression. These results suggest that targeting periostin may represent a promising strategy for overcoming CDDP resistance in NSCLC.

Some limitations of this study should be noted. First, the effect of periostin on CDDP resistance was not tested in other types of cancer cells. It is unclear whether periostinmediated CDDP resistance occurs in different types of cancers. Second, no information is available on the relationship between periostin levels and chemotherapeutic response in patients with NSCLC. Finally, the mechanism for periostin-induced activation of Stat3 is still elusive.

In conclusion, our data provide first evidence for the involvement of periostin in CDDP resistance in NSCLC cells. Periostin-mediated protection against CDDP-induced apoptosis is ascribed at least partially to activation of Stat 3 and Akt and upregulation of survivin. Periostin may represent a potential target for improving chemotherapeutic efficacy in NSCLC. 


\section{Cellular Physiology Cell Physiol Biochem 2016;38:1199-1208

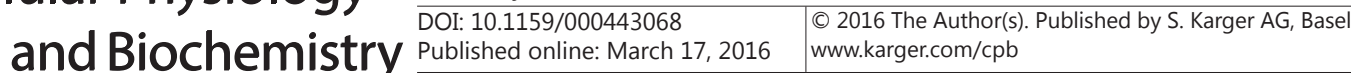 \\ Hu/Jin/Liu: Periostin Contributes to Cisplatin Resistance}

\section{Disclosure Statement}

None.

\section{References}

1 Torre LA, Bray F, Siegel RL, Ferlay J, Lortet-Tieulent J, Jemal A: Global cancer statistics, 2012. CA Cancer J Clin 2015;65:87-108.

2 Lammers PE, Lu B, Horn L, Shyr Y, Keedy V: nab-Paclitaxel in Combination With Weekly Carboplatin With Concurrent Radiotherapy in Stage III Non-Small Cell Lung Cancer. Oncologist 2015;20:491-492.

3 Florea AM, Büsselberg D: Cisplatin as an anti-tumor drug: cellular mechanisms of activity, drug resistance and induced side effects. Cancers (Basel) 2011;3:1351-1371.

4 Tian Z, Yao G, Song H, Zhou Y, Geng J: IGF2R expression is associated with the chemotherapy response and prognosis of patients with advanced NSCLC. Cell Physiol Biochem 2014;34:1578-1588.

5 Takeshita S, Kikuno R, Tezuka K, Amann E: Osteoblast-specific factor 2: cloning of a putative bone adhesion protein with homology with the insect protein fasciclin I. Biochem J 1993;294:271-278.

6 Takanami I, Abiko T, Koizumi S: Expression of periostin in patients with non-small cell lung cancer: correlation with angiogenesis and lymphangiogenesis. Int J Biol Markers 2008;23:182-186.

7 Bao S, Ouyang G, Bai X, Huang Z, Ma C, Liu M, Shao R, Anderson RM, Rich JN, Wang XF: Periostin potently promotes metastatic growth of colon cancer by augmenting cell survival via the Akt/PKB pathway. Cancer Cell 2004;5:329-339.

8 Ouyang G, Liu M, Ruan K, Song G, Mao Y, Bao S: Upregulated expression of periostin by hypoxia in nonsmall-cell lung cancer cells promotes cell survival via the Akt/PKB pathway. Cancer Lett 2009;281:213219.

9 Xiao ZM, Wang XY, Wang AM: Periostin induces chemoresistance in colon cancer cells through activation of the PI3K/Akt/survivin pathway. Biotechnol Appl Biochem 2015;62:401-406.

10 Harada D, Takigawa N, Kiura K: The Role of STAT3 in Non-Small Cell Lung Cancer. Cancers (Basel) 2014;6:708-722.

11 Kulesza DW, Carré T, Chouaib S, Kaminska B: Silencing of the transcription factor STAT3 sensitizes lung cancer cells to DNA damaging drugs, but not to TNF $\alpha$ - and NK cytotoxicity. Exp Cell Res 2013;319:506-516.

12 Chen TJ, Zhou YF, Ning JJ, Yang T, Ren H, Li Y, Zhang S, Chen MW: NBM-T-BMX-OS01, an Osthole Derivative, Sensitizes Human Lung Cancer A549 Cells to Cisplatin through AMPK-Dependent Inhibition of ERK and Akt Pathway. Cell Physiol Biochem 2015;36:893-906.

13 Zhang B, Zhang K, Liu Z, Hao F, Wang M, Li X, Yin Z, Liang H: Secreted clusterin gene silencing enhances chemosensitivity of a549 cells to cisplatin through AKT and ERK1/2 pathways in vitro. Cell Physiol Biochem 2014;33:1162-1175.

14 Kudo Y, Ogawa I, Kitajima S, Kitagawa M, Kawai H, Gaffney PM, Miyauchi M, Takata T: Periostin promotes invasion and anchorage-independent growth in the metastatic process of head and neck cancer. Cancer Res 2006;66:6928-6935.

15 Naik PK, Bozyk PD, Bentley JK, Popova AP, Birch CM, Wilke CA, Fry CD, White ES, Sisson TH, Tayob N, Carnemolla B, Orecchia P, Flaherty KR, Hershenson MB, Murray S, Martinez FJ, Moore BB; COMET Investigators: Periostin promotes fibrosis and predicts progression in patients with idiopathic pulmonary fibrosis. Am J Physiol Lung Cell Mol Physiol 2012;303:L1046-L1056.

16 Livak KJ, Schmittgen TD: Analysis of relative gene expression data using real-time quantitative PCR and the 2(-Delta Delta C(T)) Method. Methods 2001;25:402-408.

17 17.Li K, Chen B, Xu L, Feng J, Xia G, Cheng J, Wang J, Gao F, Wang X: Reversal of multidrug resistance by cisplatin-loaded magnetic Fe304 nanoparticles in A549/DDP lung cancer cells in vitro and in vivo. Int J Nanomedicine 2013;8:1867-1877.

18 Gritsko T, Williams A, Turkson J, Kaneko S, Bowman T, Huang M, Nam S, Eweis I, Diaz N, Sullivan D, Yoder S, Enkemann S, Eschrich S, Lee JH, Beam CA, Cheng J, Minton S, Muro-Cacho CA, Jove R: Persistent activation of stat3 signaling induces survivin gene expression and confers resistance to apoptosis in human breast cancer cells. Clin Cancer Res 2006;12:11-19. 


\section{Cellular Physiology Cell Physiol Biochem 2016;38:1199-1208

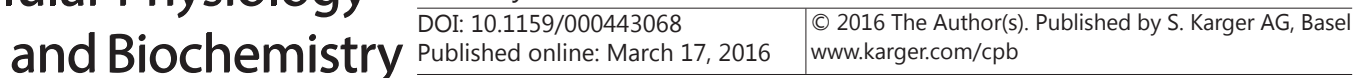 \\ Hu/Jin/Liu: Periostin Contributes to Cisplatin Resistance}

19 Schiller JH, Harrington D, Belani CP, Langer C, Sandler A, Krook J, Zhu J, Johnson DH; Eastern Cooperative Oncology Group: Comparison of four chemotherapy regimens for advanced non-small-cell lung cancer. $\mathrm{N}$ Engl J Med 2002;346:92-98.

20 Li P, Oparil S, Feng W, Chen YF: Hypoxia-responsive growth factors upregulate periostin and osteopontin expression via distinct signaling pathways in rat pulmonary arterial smooth muscle cells. J Appl Physiol (1985) 2004;97:1550-1558.

21 Schnitzer SE, Weigert A, Zhou J, Brüne B: Hypoxia enhances sphingosine kinase 2 activity and provokes sphingosine-1-phosphate-mediated chemoresistance in A549 lung cancer cells. Mol Cancer Res 2009; 7:393-401.

22 Qin S, Yang C, Wang X, Xu C, Li S, Zhang B, Ren H: Overexpression of Smac promotes Cisplatin-induced apoptosis by activating caspase- 3 and caspase- 9 in lung cancer A549 cells. Cancer Biother Radiopharm 2013;28:177-182.

23 Li B, Wang L, Chi B: Upregulation of periostin prevents P53-mediated apoptosis in SGC-7901 gastric cancer cells. Mol Biol Rep 2013;40:1677-1683.

24 Zhang Z, Nie F, Chen X, Qin Z, Kang C, Chen B, Ma J, Pan B, Ma Y: Upregulated periostin promotes angiogenesis in keloids through activation of the ERK $1 / 2$ and focal adhesion kinase pathways, as well as the upregulated expression of VEGF and angiopoietin-1. Mol Med Rep 2015;11:857-864.

25 Yang L, Serada S, Fujimoto M, Terao M, Kotobuki Y, Kitaba S, Matsui S, Kudo A, Naka T, Murota H, Katayama I: Periostin facilitates skin sclerosis via PI3K/Akt dependent mechanism in a mouse model of scleroderma. PLoS One 2012;7:e41994.

26 Hu Y, Hong Y, Xu Y, Liu P, Guo DH, Chen Y: Inhibition of the JAK/STAT pathway with ruxolitinib overcomes cisplatin resistance in non-small-cell lung cancer NSCLC. Apoptosis 2014;19:1627-1636.

27 Zhu M, Saxton RE, Ramos L, Chang DD, Karlan BY, Gasson JC, Slamon DJ: Neutralizing monoclonal antibody to periostin inhibits ovarian tumor growth and metastasis. Mol Cancer Ther 2011;10:1500-1508.

28 Kyutoku M, Taniyama Y, Katsuragi N, Shimizu H, Kunugiza Y, Iekushi K, Koibuchi N, Sanada F, Oshita Y, Morishita R: Role of periostin in cancer progression and metastasis: inhibition of breast cancer progression and metastasis by anti-periostin antibody in a murine model. Int J Mol Med 2011;28:181-186. 